\title{
Transmission of the Pabl family of restriction DNA glycosylase genes: mobility and long-term inheritance
}

Kenji K. Kojima ${ }^{1,2,3}$ and Ichizo Kobayashi ${ }^{1,2^{*}}$

\begin{abstract}
Background: R.Pabl is an exceptional restriction enzyme that functions as a DNA glycosylase. The enzyme excises an unmethylated base from its recognition sequence to generate apurinic/apyrimidinic (AP) sites, and also displays AP lyase activity, cleaving the DNA backbone at the AP site to generate the $3^{\prime}$-phospho alpha, beta-unsaturated aldehyde end in addition to the 5'-phosphate end. The resulting ends are difficult to religate with DNA ligase. The enzyme was originally isolated in Pyrococcus, a hyperthermophilic archaeon, and additional homologs subsequently identified in the epsilon class of the Gram-negative bacterial phylum Proteobacteria, such as Helicobacter pylori.

Results: Systematic analysis of R.Pabl homologs and their neighboring genes in sequenced genomes revealed co-occurrence of R.Pabl with M.Pabl homolog methyltransferase genes. R.Pabl and M.Pabl homolog genes are occasionally found at corresponding (orthologous) loci in different species, such as Helicobacter pylori, Helicobacter acinonychis and Helicobacter cetorum, indicating long-term maintenance of the gene pair. One R.Pabl and M.Pabl homolog gene pair is observed immediately after the GMP synthase gene in both Campylobacter and Helicobacter, representing orthologs beyond genera. The mobility of the Pabl family of restriction-modification (RM) system between genomes is evident upon comparison of genomes of sibling strains/species. Analysis of R.Pabl and M.Pabl homologs in H. pylori revealed an insertion of integrative and conjugative elements (ICE), and replacement with a gene of unknown function that may specify a membrane-associated toxin (hrgC). In view of the similarity of HrgC with toxins in type I toxin-antitoxin systems, we addressed the biological significance of this substitution. Our data indicate that replacement with hrgC occurred in the common ancestor of hspAmerind and hspEAsia. Subsequently, H. pylori with and without $h r g C$ were intermixed at this locus, leading to complex distribution of hrgC in East Asia and the Americas. In Malaysia, hrgC was horizontally transferred from hspEAsia to hpAsia2 strains.

Conclusions: The Pabl family of RM system behaves as a mobile, selfish genetic element, similar to the other families of Type II RM systems. Our analysis additionally revealed some cases of long-term inheritance. The distribution of the hrgC gene replacing the Pabl family in the subpopulations of H. pylori, hspAmerind, hspEAsia and hpAsia2, corresponds to the two human migration events, one from East Asia to Americas and the other from China to Malaysia.
\end{abstract}

Keywords: Pabl, Restriction endonuclease, Restriction-modification system, DNA glycosylase, DNA methylation, Methyltransferase, Helicobacter pylori, hrgC

\footnotetext{
* Correspondence: ikobaya@ims.u-tokyo.ac.jp

'Department of Computational Biology and Medical Sciences, Graduate

School of Frontier Sciences, University of Tokyo, Minato-ku, Tokyo 108-8639,

Japan

${ }^{2}$ Institute of Medical Science, University of Tokyo, Minato-ku, Tokyo 108-8639,

Japan

Full list of author information is available at the end of the article
}

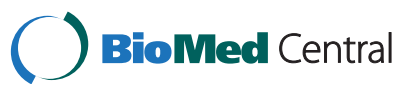

(C) 2015 Kojima and Kobayashi. Open Access This article is distributed under the terms of the Creative Commons Attribution 4.0 International License (http://creativecommons.org/licenses/by/4.0/), which permits unrestricted use, distribution, and reproduction in any medium, provided you give appropriate credit to the original author(s) and the source, provide a link to the Creative Commons license, and indicate if changes were made. The Creative Commons Public Domain Dedication waiver (http://creativecommons.org/publicdomain/zero/1.0/) applies to the data made available in this article, unless otherwise stated. 


\section{Background}

Restriction enzymes represent a group of sequencespecific endonucleases that cleave DNA of a specific epigenetic modification state [1], resulting in defense against selfish genetic elements, such as viruses (bacteriophages) and plasmids. A restriction enzyme (R) gene is usually linked with a modification enzyme $(\mathrm{M})$ gene that recognizes the same target sequence and methylates it to prevent cleavage of DNA by the restriction enzyme. Several studies have reported that some restriction-modification (RM) systems are also selfish genetic elements, killing cells that have lost them through chromosome cleavage $[2,3]$. Such post-segregational killing is commonly observed with RM systems classified as 'Type II'. Bioinformatics, molecular evolutionary analyses and laboratory experiments have validated their behavior as mobile genetic elements that insert into and rearrange genomes.

A type II restriction enzyme, R.PabI [4, 5] exhibiting a "half-pipe" fold [6], has been identified as a DNA glycosylase that excises the adenine base from its recognition sequence, GTAC, when not methylated at this site to generate an apurinic/apyrimidinic (AP) site [7, 8]. R.PabI has also been shown to exert AP lyase activity [8]. Until these findings, all restriction enzymes examined had been identified as DNA phosphodiesterases [9]. Despite different ternary structures, all these enzymes cleave the sugar-phosphate backbone of DNA, leaving 5'-phosphate and 3'-OH ends. The AP lyase activity of R.PabI generates a different terminal structure, 5'-phosphate and 3'-phospho alpha, beta-unsaturated aldehyde (3'-PUA) ends [8], which are difficult to re-ligate via DNA ligase. These results led to the reclassification of restriction enzymes into restriction phosphodiesterase and glycosylase subgroups [7, 8].

R.PabI was originally identified from a hyperthermophilic archaeon Pyrococcus abyssi during genome comparison with Pyrococcus horikoshii $[4,5]$. A region including the R.PabI gene and the neighboring methyltransferase gene encoding a DNA methyltransferase, M.PabI, is present in the genome of $P$. abyssi, but absent at the orthologous locus in the genome of $P$. horikoshii [10], which contains an $18 \mathrm{~kb}$ sequence instead [5]. Homologs of R.PabI have been detected in various archaea and bacteria, with particular abundance in Epsilonproteobacteria, such as Helicobacter pylori [7]. The R.PabI homolog in H. pylori, termed R.HpyAXII, is reported to be a GTAC-specific restriction enzyme [11]. The neighboring gene encodes M.HpyAXII, an adenine methyltransferase. These findings have led to the proposal that R.PabI and M.PabI homolog pairs constitute an RM system targeting GTAC tetranucleotides. However, systematic evolutionary analysis of the R.PabI and M.PabI homologs has not been performed to date.

H. pylori, a Gram-negative pathogenic bacterium colonizing the stomach, is associated with several gastrointestinal disorders [12]. H. pylori is highly divergent in genome sequences within the species [13]. Due to stable transmission from parents to children, the lineages of $H$. pylori maintain traces of human migration. This characteristic was used to reveal human migration to the Americas and Pacific islands [14, 15]. At present, based on the sequences of seven conserved genes, $H$. pylori is classified into several populations (hpAfrica2, hpAfrica1, hpNEAfrica, hpEurope, hpSahul, hpAsia2, and hpEastAsia), each with a distinct geographical distribution $[12,15,16]$. These are further grouped into subpopulations. For example, hpEastAsia is subdivided into hspEAsia, hspMaori, and hspAmerind, consistent with the East Asian origin of Amerind (native American) people [15]. Among these, hspEAsia and hspAmerind are further divided into subgroups [17].

In the current study, systematic examination revealed clear evidence of co-insertion of R.PabI and M.PabI homolog genes. R.PabI and M.PabI homologs in various strains of $H$. pylori and related species, $H$. acinonychis and $H$. cetorum, were orthologous, signifying insertion in the common ancestor. Two examples of cross-genus presence of R.PabI and M.PabI pair at the orthologous locus are also found. Moreover, we showed the geographic and phylogenetic distribution of the $h r g C$ gene replacing R.PabI and M.PabI homolog genes in H. pylori, and discussed its implications in human migration.

\section{Results}

\section{Co-occurrence of R.Pabl and M.Pabl homologs}

R.PabI homologs have been identified from various species of archaea and bacteria [7]. Our extensive PSI-BLAST search led to the identification of several homologs from various species. R.PabI homologs were identified for the first time for Brachyspira sp., Mycoplasma primatum, Mucispirillum schaedleri, as well as several species from the genera Campylobacter and Helicobacter (Additional file 1: Table S1; Additional file 2: Table S2). Analysis of the gene neighborhood of R.PabI homologs showed that almost all R.PabI homologs are next to an adenine methyltransferase gene (Additional file 1: Table S1; Additional file 2: Table S2), as reported for P. abyssi and H. pylori $[5,10,11]$.

Phylogenetic trees were compared based on R.PabI homologs and neighboring methyltransferase genes (Fig. 1). Due to frequent disruption in R.PabI homologs, compared to that in neighboring methyltransferase genes, Fig. 1a includes fewer sequences than Fig. 1b. Hereafter, these methyltransferase genes are designated 'M.PabI homologs'. BLASTP search with M.PabI as a query led to the identification of several homologous protein sequences that are not associated with R.PabI homologs (data not shown). Using an M.PabI homolog from $H$. pylori as a query, BLASTP hits were mainly proteins associated with R.PabI homologs, and archaeal M.PabI 
a R.Pabl homolog

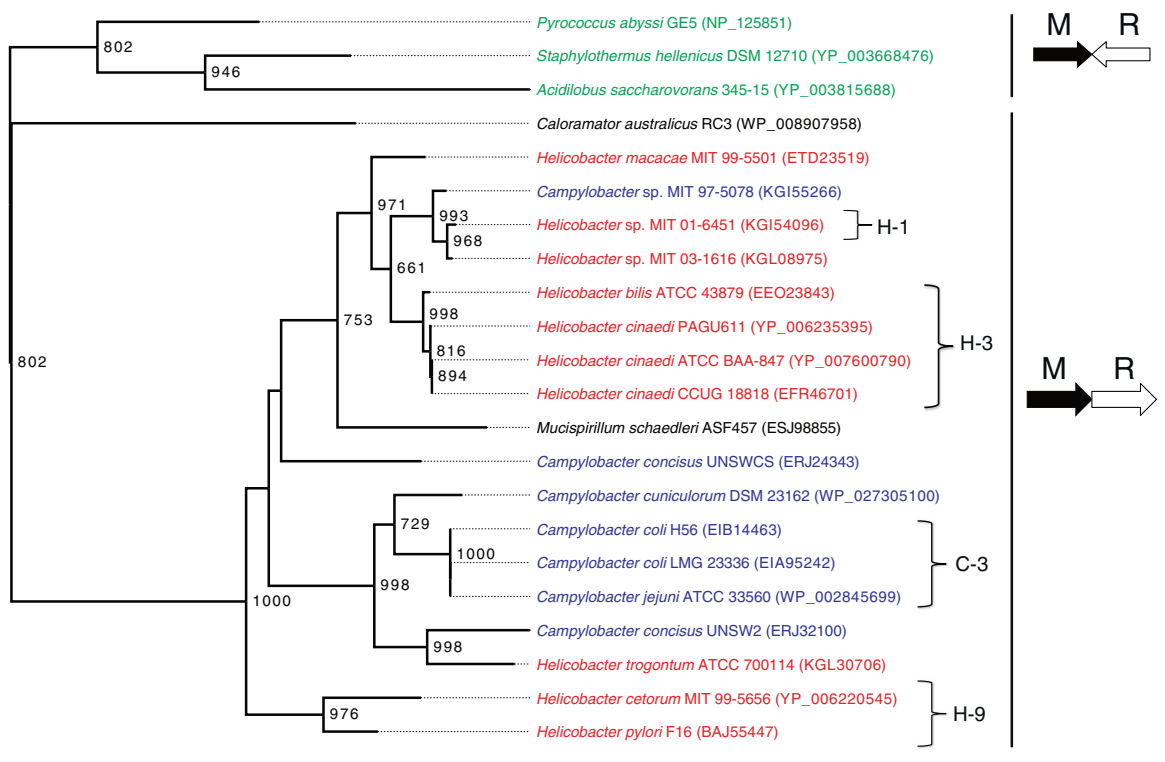

0.4

b M.Pabl homolog

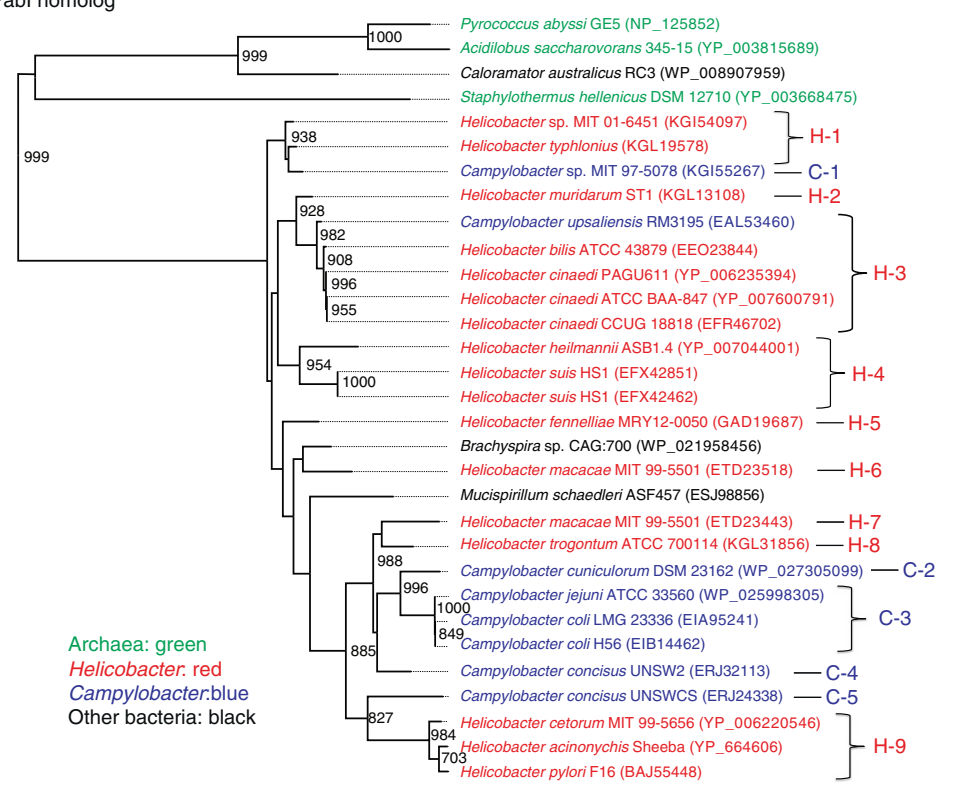

$\overline{0.7}$

Fig. 1 Phylogeny of R.Pabl and M.Pabl homologs. Trees are rooted at the midpoint. Bootstrap values of 1000 replicates over $50 \%$ are shown at the nodes. Archaeal sequences are colored in green, Helicobacter sequences in red and Campylobacter sequences in blue. a Tree of R.Pabl homologs. Four orthologous loci $(\mathrm{H}-1, \mathrm{H}-3, \mathrm{C}-3$, and $\mathrm{H}-9)$ are indicated on the right. The order of R.Pabl and M.Pabl homolog genes is illustrated on the far right. $\mathbf{b}$ Tree of M.Pabl homologs. Loci are indicated on the right: $\mathrm{H}-1$ to $\mathrm{H}-9$ for Helicobacter and $\mathrm{C}-1$ to $\mathrm{C}-5$ for Campylobacter. Neighboring genes for each locus are shown in Table 1

homologs from Acidilobus and Pyrococcus were hit with e-values of 0.002 and 0.004 , respectively. Both archaeal and bacterial M.PabI homologs are gamma-type methyltransferases containing a target recognition domain (TRD) at the C-terminus. Bacterial M.PabI homologs are shorter than their archaeal counterparts due to the shorter C-terminal regions.

Both trees showed two lineages nearly corresponding to archaea and bacteria. Two closely related Epsilonproteobacteria genera, Campylobacter and Helicobacter, 
contained almost all the bacterial PabI homologs. It is likely that PabI homologs in Mucispirillum schaedleri (Mollicutes) and Brachyspira sp. (Spirochaetes) were horizontally transferred from Epsilonproteobacteria. The position of Caloramator (Firmicute, Bacteria) was not consistent between the phylogenies of M.PabI and R.PabI homologs, and may represent another lineage distant from archaeal and bacterial lineages or recombination of R.PabI of one subfamily and M.PabI of another (see next paragraph).

The gene order in the PabI family of RM systems corresponded to the phylogeny of M.PabI and R.PabI homologs. The archaeal lineage contained the $M$ and $R$ genes in a tail-to-tail orientation, while the bacterial lineage contained the $\mathrm{M}$ gene upstream of the $\mathrm{R}$ gene in the same orientation. This finding raises the possibility that archaeal and bacterial M.PabI homologs are not orthologous, and represent two lineages independently paired with R.PabI homologs.

\section{M.Pabl and R.Pabl homologs at orthologous sites shared by species}

Comparison of Helicobacter and Campylobacter genomes revealed at least $9(\mathrm{H}-1$ to $\mathrm{H}-9)$ and $5(\mathrm{C}-1$ to $\mathrm{C}-5)$ loci for the PabI family of RM systems (Fig. 1 and Table 1). Some of these loci are shared by different species $(\mathrm{H}-1, \mathrm{H}-3$, $\mathrm{H}-4, \mathrm{H}-9, \mathrm{C}-3)$ or genera ( $\mathrm{H}-3$ and $\mathrm{C}-1, \mathrm{H}-2$ and $\mathrm{C}-4)$ as detailed below. To confirm the orthology of these crossspecies/genera RM systems, we aligned the spacer sequences between the upstream gene and the $M$ gene (Additional file 3: Figure S1), and found that they are very likely orthologous. Campylobacter upsaliensis RM 3195 has an M.PabI homolog similar to M.PabI homologs at the H-3 locus in Helicobacter (Fig. 1). It was revealed to have been transferred horizontally from Helicobacter and thus we included this M.PabI homolog in the H-3 lineage even though it was from Campylobacter. The details are described in the next section.

Three closely related M.PabI and R.PabI homologs from two Campylobacter coli strains (LMG 23336 and H56) and C. jejuni strain ATCC 33560 (C-3 in Fig. 1) were located at the orthologous locus (Additional file 3: Figure S1). However one lineage of $C$. coli (clade I) is reported to have experienced massive introgression from C. jejuni, and both $C$. coli strains for C-3 belong to clade I [18]. In fact, no other strains of $C$. coli contained the insertion of the PabI family (data not shown). In view of these results, it is unlikely that insertion of PabI family is an ancestral feature shared by $C$. coli and C. jejuni.

Among the $H$. pylori strains, M.PabI and R.PabI homolog gene pairs were similar. The amino acid identities of M.PabI and R.PabI homologs in 599 (hpWAfrica) and F16 (hspEAsia) were 317/342 (93 \%) and 228/251 (91 \%), respectively. In $H$. pylori, the M.PabI and R.PabI homolog gene pair was located between DNA gyrase B $($ gyrB $)$ and an outer membrane protein gene represented by HP0506/HP0507 in strain JHP0457 in strain/JHP0457 in strain $\mathrm{J99}$ (Fig. 2a).

The only exception was ELS37 containing two R.PabI homologs, of which one was severely truncated. Their protein sequences were identical in the alignable region. The R.PabI homolog next to M.PabI homolog was intact. Comparison with genomes of the $H$. pylori strains, NY40 and P12, revealed a 1577 bp duplication, coupled with insertion of a $\sim 50 \mathrm{~kb}$ mobile genetic element (Fig. 2b). If this insertion was excluded, the PabI family was located

Table 1 Loci for the Pabl family of RM systems in Helicobacter and Campylobacter

\begin{tabular}{|c|c|c|}
\hline Locus & Upstream gene & Downstream gene \\
\hline $\mathrm{H}-1$ & Hypothetical protein containing ankyrin repeats & Multidrug transporter MatE/RelE-family toxin \\
\hline $\mathrm{H}-2$ & Adenylosuccinate lyase & Hypothetical protein \\
\hline $\mathrm{H}-3$ & GMP synthase & Poly A polymerase/Methionyl-tRNA formyltransferase \\
\hline $\mathrm{H}-4$ & Shikimate 5-dehydrogenase & DNA polymerase III subunit delta/Hypothetical protein \\
\hline $\mathrm{H}-5$ & Glutamate synthase large chain & Glutamate synthase small chain \\
\hline $\mathrm{H}-6$ & ABC transporter permease & TetR family transcriptional regulator \\
\hline $\mathrm{H}-7$ & ABC transporter ATP-binding protein & MFS transporter \\
\hline $\mathrm{H}-8$ & Argininosuccinate synthase & Long-chain fatty acid transport protein \\
\hline$H-9$ & DNA gyrase B & Outer membrane protein 8/Outer membrane protein \\
\hline$C-1$ & GMP synthase & (No sequence information) \\
\hline$C-2$ & Imidazole glycerol-phosphate dehydratase & Imidazole glycerol phosphate synthase \\
\hline$C-3$ & Carbamoyl phosphate synthase large subunit & COG0009 Putative translation factor (SUA5) \\
\hline$C-4$ & Adenylosuccinate lyase & Ribonucleotide reductase of class la (aerobic), alpha subunit \\
\hline$C-5$ & RelE family toxin & Hypothetical protein with DUF4279 \\
\hline
\end{tabular}

The closest genes that are not components of RM system are shown. In cases of more than two gene arrangements in the same group, two genes are shown, divided by a slash (/). H-1 to H-9 are in Helicobacter, C-1 to C-5 in Campylobacter. Orthologous genes seen in different locus groups are italicized (H-2, H-3, C-1 and C-4) 


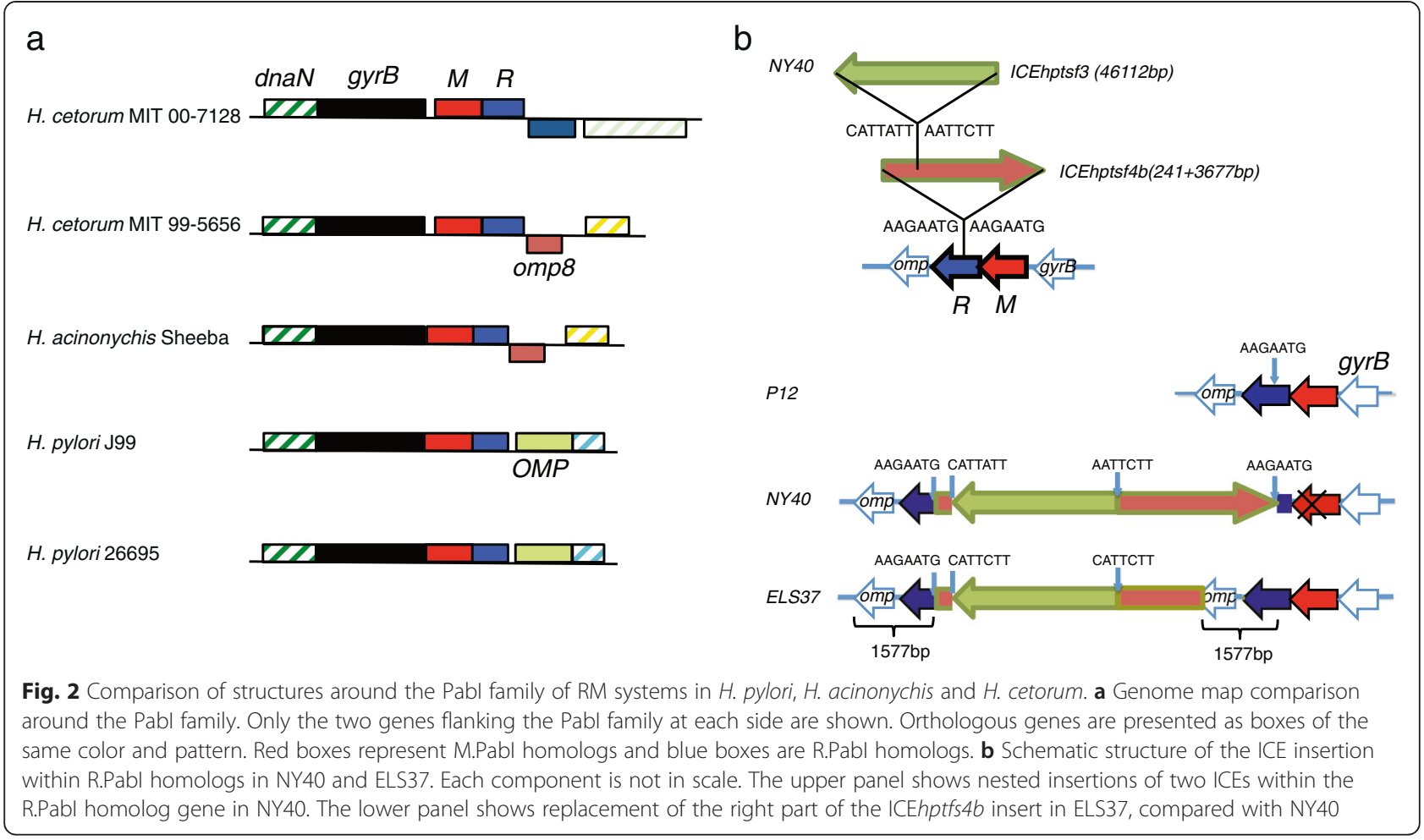

at the same site as in other $H$. pylori strains. This mobile genetic element was recently characterized as two integrative and conjugative elements (ICE), designated ICEhptfs3 and ICEhptsf4b [19]. In the genome of NY40, ICEhptfs $4 b$ was inserted within the R.PabI homolog, duplicating AAGAATG, and ICEhptfs3 within the ICEhptfs $4 B$ copy, with AAGAATG target site duplication in the opposite orientation. Duplicated sequences were mutated in NY40 to AAGAAT $\underline{T}$ and AATAATG. Here, the R.PabI homolog gene was split into two segments, and the M.PabI homolog gene disrupted by mutations. In the ELS37 genome, the 1654 bp right terminal region of ICEhptsf $4 b$ was replaced by a duplicated 1577 bp segment containing part of the R.PabI homolog gene and part of an outer membrane protein gene. Thus, this duplication possibly occurred via a gene conversion-like mechanism with incoming DNA of intact R.PabI homo$\log$ as the template. The right junction appeared to result in homologous recombination and the left junction in non-homologous recombination. This recombination event resulted in a 1577 bp duplication sandwiching the $\sim 50 \mathrm{~kb}$ ICE insertion.

In the genomes of $H$. acinonychis and $H$. cetorum, two species closely related to $H$. pylori, the M.PabI and R.PabI homolog gene pair was located downstream of gyrB (Fig. 2a). The gene downstream of the PabI homolog gene pair was outer membrane protein 8 (omp8), which is absent in $H$. pylori. The intergenic sequence between $g y r B$ and the M.PabI homolog was shorter than
$10 \mathrm{bp}$ and appeared conserved, except for $H$. cetorum MIT 99-5656, which contained a sequence of unknown origin between the two genes (Additional file 3: Figure S1). Among these species, $H$. pylori and $H$. acinonychis are reported to be close, while $H$. cetorum is more divergent [20]. Data from our phylogenetic analysis of M.PabI homologs were consistent with this relationship (Fig. 1). The most parsimonious explanation is that the PabI family was integrated between gyrB and omp8 in the common ancestor of $H$. pylori, $H$. acinonychis and $H$. cetorum, and the downstream region rearranged. The results collectively suggest that the PabI family has been maintained for a long time in these species. Linkage of RM systems with genome rearrangement junctions has been reported [21].

\section{M.Pabl and R.Pabl homologs at orthologous sites shared by genera}

$\mathrm{H}-3$ in the genus Helicobacter and C-1 in Campylobacter occur downstream of a homologous gene for GMP synthase (Table 1). Although GMP synthase in C-1 corresponds to only a C-terminal fragment of the complete protein, the two GMP synthase proteins are $88 \%$ identical in the alignable region. The junctions between the genes for GMP synthase and M.PabI homolog in $\mathrm{H}-3$ and $\mathrm{C}-1$ resembled each other to a significant extent (Additional file 3: Figure S1). A sequence almost identical to the junction sequence in H-3 was found in C. upsaliensis RM3195, followed by a gene for the M.PabI homolog 
(Fig. 1b, Additional file 3: Figure S1). This M.PabI homo$\log$ (EAL53460) displayed $89 \%$ amino acid sequence identity to that from $H$. cinaedi PAGU611. This M.PabI homolog is encoded near a contig end, and the partner R.PabI homolog is not on the contig. Instead, we identified a fragmented R.PabI homolog (EAL52983) coded at the end of another contig (Table S1). This R.PabI homolog is $\sim 96 \%$ identical to those in $H$. cinaedi and $\sim 93 \%$ to that in $H$. bilis. Therefore it is very likely that EAL53460 and EAL52983 are an M.PabI and R.PabI homolog pair.

Other C. upsaliensis strains, JV21 and DSM 5365, lacked the M.PabI and R.PabI homolog pair, but their sequences for GMP synthase were more similar to those of RM3195 and some Helicobacter species than those of other Campylobacter species. It indicates that the GMP synthase gene was horizontally transferred from Helicobacter to the common ancestor of C. upsaliensis. In the three strains of C. upsaliensis, the GMP synthase gene is followed by a non-homologous gene, indicating genome rearrangement downstream of the GMP synthase gene. A single event of horizontal transfer of the GMP synthase gene as well as the PabI family and subsequence recombination leading to the loss of the PabI family in some strains is more likely, compared with two independent horizontal transfer events (the GMP synthase gene and the PabI family) to the same genomic locus. Thus, we conclude that the H-3 lineage is horizontally transferred from Helicobacter to C. upsaliensis with the flanking GMP synthase gene.

The origin of insertion at C-1 in Campylobacter sp. MIT 97-5078 is unknown at present. One possibility is that the PabI family was present in the common ancestor of Campylobacter and Helicobacter, and has been lost in many species/strains. We cannot eliminate another horizontal transfer between Helicobacter and Campylobacter, but even so, the transfer must be very old, considering their sequence diversity. Long-term maintenance of the PabI family at the orthologous locus is the most likely scenario.

Similarly, two adenylosuccinate lyase proteins encoded upstream of C-4 and $\mathrm{H}-2$ displayed $73 \%$ identity. The genome of $H$. muridarum have $6 \mathrm{M}$ (pseudo)genes and 4. $\mathrm{R}$ (pseudo)genes between two non-RM genes there (Additional file 4: Figure S2). Here, recurrent acquisition of different RM systems has obscured the relationship between $\mathrm{C}-4$ and H-2 (Additional file 3: Figure S1), but we cannot exclude the orthology of these two loci.

\section{Gene neighborhood of the Pabl family of RM systems}

In $\mathrm{H}-5$ and $\mathrm{C}-2$, PabI family is located in a putative operon whose genes are tightly linked in function and encoded on the same strand (Table 1). Glutamate synthase large chain and small chain are components of the same enzyme for glutamate metabolism. Both imidazole glycerol phosphate dehydratase and imidazole glycerol phosphate synthase play a role in histidine metabolism. In these cases, the RM system is coded on the same strand as the neighboring genes. This type of operon insertion has been reported for other RM systems and operons [22], and may impose operon maintenance and expression through post-segregational killing.

The PabI family is occasionally linked with other RM or toxin-antitoxin systems. The genes encoding defense system components, such as RM, toxin-antitoxin, and CRISPR-Cas systems typically cluster in "defense islands" [23]. The PabI family in H. cinaedi PAGU611 is flanked by an RM system. The PabI family in C. cuniculorum DSM 23162 replaced a restriction enzyme gene (see next section for details). The extreme case is observed in $H$. muridarum (Additional file 4: Figure S2). Between two non-RM genes, there are $6 \mathrm{M}$ (pseudo)genes and $4 \mathrm{R}$ (pseudo)genes. The PabI family of $\mathrm{H}-1$ and $\mathrm{C}-5$ is flanked by genes for the RelE toxin family. These two toxin genes show very weak sequence similarity to each other (data not shown), indicating they represent independent association of RM system and RelE toxin genes.

\section{Insertion of Pabl family of RM system}

Genome sequence comparison among closely related species/strains revealed the mobility of the PabI family (Fig. 3). Comparison of Staphylothermus hellenicus DSM 12710 and S. marinus F1 revealed insertion into S. hellenicus of three ORFs: R.PabI and M.PabI homologs and part of a penicillinase repressor (Fig. 3a). This PabI RM system replaced a 62 bp DNA segment.

Comparison of Campylobacter coli LMG 23336 (locus C-3) and 15-537360 strains disclosed a sequence insertion only including two genes for R.PabI and M.PabI homologs (Fig. 3b). This RM system replaced the $4 \mathrm{bp}$ sequence, AGCT. All C. coli strains available at the NCBI BLAST website other than LMG 23336 and H56 displayed a conserved structure identical to 15-537360. TAAA tetranucleotides were observed at the insertion boundary, although it is unclear whether they represent target site duplications.

Comparison of Campylobacter cuniculorum DSM23162 (locus C-2) and C. upsaliensis RM3195 revealed replacement of a putative Type II RM system R gene with a PabI family (Fig. 3c). The (presumably cognate) $M$ gene neighboring the replaced $\mathrm{R}$ gene and flanking $h r g A$ gene were disrupted in the DSM23162 genome. The $h r g A$ gene was previously reported to replace a restriction enzyme gene in H. pylori [24], but the $h r g A$ gene seen here does not replace an RM system. The putative Type II RM system itself was possibly inserted in the ancestral locus of these two genomes, since Campylobacter coli 111-3 lacks the Type II RM system. According to REBASE 
a

Staphylothermus marinus F1

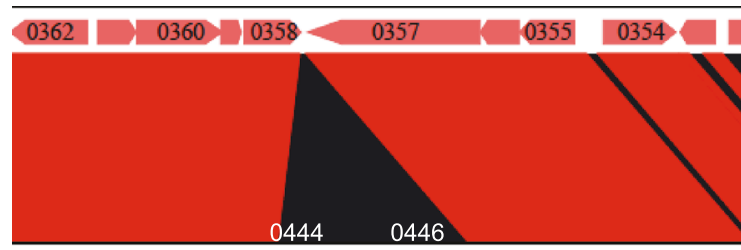

$439 \square 0441>0443$ 口 $0445 \times 0447 \quad 0449$

Staphylothermus hellenicus DSM 12710
Shell_0444 Penicillinase repressor

Shell_0445 M.Pabl homolog

Shell_0446 R.Pabl homolog
F1
GATCATGGCTTCATAAGGTTTGTTAGAGATTTATGAAACG (
111111111111111111
22bP) TAATTTATAGAAGAAGGAAGATATAGAGAAAGAAA---AT
DSM 12710 GATCATGGTTTTATAAGGTTAGCTAAATTTTAAATGAGTT (2634bp) TTAGCTATAACCATAGTCAAATATAGAGAAAGAAAGAGAA

b

Campylobacter coli LMG 23336

69636968

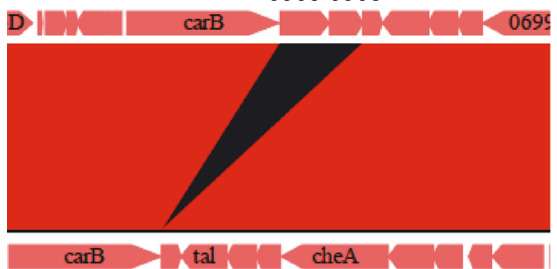

cco76 06963 M.Pabl homolog

cco76_06968 R.Pabl homolog

Campylobacter coli 15-537360

LMG 23336 AAGAGTTTGCAAGAATATTTAAAGGACTAGTATTGATGCAAAA (1748bp) AGTTTATTTTAAATAGTAAATGATTTATCTTGCACAAACC

II II IIII II IIIIIIII

1111111111 | | |1111

$15-537360$

TTGATTATCTAGCGCAAACA

C

Campylobacter cuniculorum DSM 23162

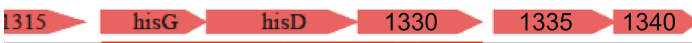

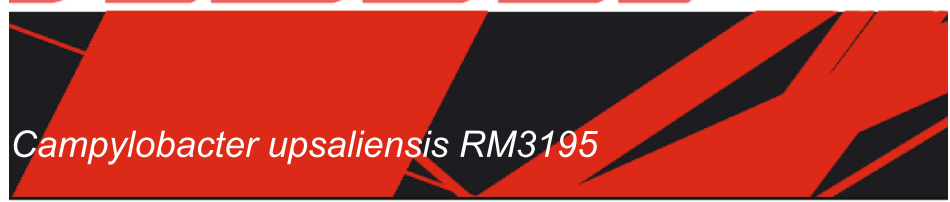

Q330_RS0101335 M.Pabl homolog Q330_RS0101340 R.Pabl homolog

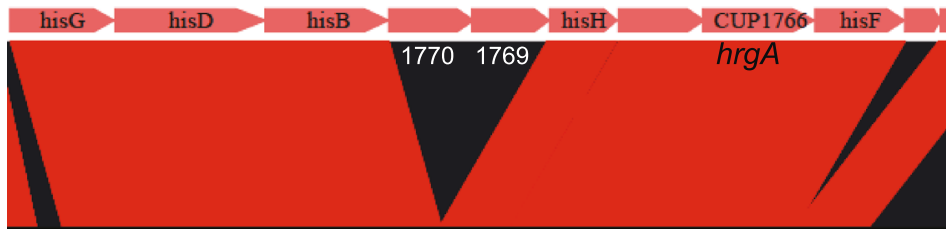

CUP1770 type II restriction enzyme

CUP1769 adenine-specific

DNA methyltranserase

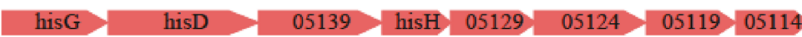

Campylobacter coli 111-3

DSM 23162 GCTCTAAAGGTGGATATGAATTATTTTTAGAAGTCAAGG (2289bp) AAAATAAAAATGCCCCTTATACTTTTAACTATAAAACTAT

RM3195 GTTCTAAGGGGTGTTATGACAAAAATAGAAGCTATTGAA( 703bp) GTATAAAAAATACAAAATTCACTTTTAATATAAAACAAT

Fig. 3 (See legend on next page.) 
(See figure on previous page.)

Fig. 3 Integration of the Pabl family of RM systems. Two genome maps are compared. Red boxed arrows indicate genes with coding directions. Gene numbers or names are presented in or near the arrows. Regions of sequence similarity between loci are indicated by red bands. Junction sequences are shown below the genome map comparison. Genes within the insertions with their annotations are shown on the right. a Genome map comparison of Staphylothermus marinus F1 and S. hellenicus DSM 12710 around the Pabl family in the S. hellenicus genome. b Genome map comparison of Campylobacter coli LMG 23336 and C. coli 15-537360 around the Pabl family in the LMG 23336 genome. c Genome map comparison of Campylobacter cuniculorum DSM 23162, C. upsaliensis RM3195 and C. coli 111-3 around the Pabl family in the DSM 23162 genome

(http://tools.neb.com/blast/), this putative RM system recognizes 5'GANTC.

\section{Identification of two members of the Pabl family of RM system in $H$. macacae MIT 99-5501}

We identified two PabI family members from $H$. macacae MIT 99-5501 (Table 1 and Fig. 1, loci H-6 and H-7). One R.PabI homolog (annotated as two proteins, ETD23441 and ETD23442) was disrupted, but the other R.PabI homolog (ETD23519) and both M.PabI homologs (ETD23443 and ETD23518) appeared intact. Phylogenetic analysis (Fig. 1) indicated that these two members of the PabI family were independently acquired by $H$. macacae. One M.PabI homolog (ETD23443), associated with a disrupted R.PabI homolog, was most closely related to that from Helicobacter trogontum (KGL31856), and the other (ETD23518) neighboring an intact R.PabI homolog (ETD23519) related to that from Brachyspira (WP_021958456).

\section{Shared rearrangement in the Pabl family of RM system in hspEAsia and hspAmerind}

Some R.PabI homolog genes in $H$. pylori are disrupted due to frameshift, nonsense mutations, and/or deletions (data not shown). All hspAmerind strains sequenced completely to date contain a large deletion within the PabI family (Fig. 4a). The same deletion is observed in some hspEAsia strains, such as 52 (Korea), 83, F30 (Fukui, Japan) and OK310 (Okinawa, Japan). Sequence comparisons revealed that these deletions are caused by replacement with a DNA segment containing one gene, annotated $\operatorname{hrgC}$ (Fig. 4b). While this substitution was previously reported [11], its geographical distribution remains to be established. Among the complete genomes for $H$. pylori, only hspAmerind and hspEAsia strains contained hrgC (Fig. 4a), placed in an opposite orientation to the M.PabI and R.PabI homologs. hspEAsia is subdivided into 4 subgroups in Yahara et al. [17], and no subgroup show uniform presence/absence of $h r g C$ (Fig. 4c).

The $h r g C$ gene encodes a small protein with a length of $<100$ residues (Fig. 4d). Although the function of $\mathrm{hrgC}$ gene has not been confirmed, its homologs have been identified in various bacteria, including Escherichia coli and Bacillus cereus, indicating horizontal transfer of the gene. Secondary structure prediction with jpred3 (http:// www.compbio.dundee.ac.uk/www-jpred/) [25] indicated that the HrgC protein contains one transmembrane helix. This result is consistent with the annotation of some HrgC homologs as inner membrane proteins. These features are found in toxins of type I toxin-antitoxin systems [26], leading to the speculation that $\mathrm{HrgC}$ is a toxin of a toxin-antitoxin system (see Discussion).

The 5'-terminal 78 bp M.PabI and 3'-terminal 88 bp R.PabI homolog genes were observed at the locus in these $h r g C$-encoding strains, indicating that the PabI family is replaced by the $h r g C$ gene and not vice versa (Additional File 5: Figure S3).

$h r g C$ was also located between $d n a K$ and $c y s K$ in the genome of Helicobacter acinonychis Sheeba as well as several $H$. pylori strains, such as G27 (Fig. 4b, d). This finding is in keeping with the report that $H$. acinonychis and $H$. pylori strain NSH57 (a derivative of G27) contain the hrgC gene at a locus distinct from the "pabl" locus [11]. The locus is occupied by a gene that encodes a protein similar to the N-terminal region of McrB in most $H$. pylori strains. McrB is a DNA binding subunit of Type IV (methylationspecific) restriction endonuclease that acts in concert with McrC [22, 27]. Notably, the 166 bp 5' fragment of the M.PabI gene was associated with the $h r g C$ gene at the " $m c r B$ " locus (Additional file 5: Figure S3). Compared with HrgC proteins encoded at the "pabI" locus, those encoded at the " $m c r B$ " locus lacked a $\sim 35$ aa segment (Fig. 4d, G27 and Sheeba). This difference may be due to an insertion in the lineage at the "pabl" locus, since $\mathrm{HrgC}$ proteins encoded within the genomes of other species do not contain a sequence corresponding to this 35 aa segment. Thus, insertion of 35 aa segment is likely a derived feature.

The TBLASTN search with $\mathrm{HrgC}$ protein as a query against the Whole genome shotgun sequence (wgs) database led to the identification of several more $H$. pylori strains containing the $h r g C$ gene. These sequences were encoded at either the "pabl" or " $m c r B$ " locus (data not shown). Interestingly, HrgC proteins encoded at the "pabl" locus in some strains lacked the $\sim 35$ aa segment (Fig. 4d, oki102 and Hp A-26), including four strains (oki102, oki112, oki422 and oki898) from Okinawa, Japan, three from North America (R056a, R018c and Hp A-26), 173/00 from Portugal, and A45 from Russia. In summary, HrgC proteins with the $\sim 35$ aa segment have been identified from Japan, Korea, China, Malaysia, and South America (Fig. 4e). 


\begin{tabular}{|c|c|c|c|}
\hline \multicolumn{4}{|c|}{ 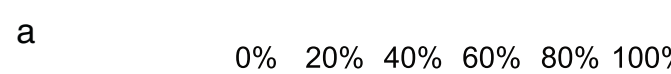 } \\
\hline \multicolumn{2}{|c|}{ hpAfrica2 } & \multicolumn{2}{|l|}{2} \\
\hline \multicolumn{2}{|c|}{ hpAfrica1 } & \multicolumn{2}{|l|}{6} \\
\hline \multicolumn{2}{|c|}{ hpEurope } & \multicolumn{2}{|l|}{14} \\
\hline \multicolumn{2}{|c|}{ hpAsia2 } & \multicolumn{2}{|l|}{2} \\
\hline \multicolumn{2}{|c|}{ hspEAsia } & 11 & 4 \\
\hline \multicolumn{2}{|c|}{ hspAmerind } & \\
\hline & & Pabl & $\square \mathrm{hrgC}$ \\
\hline \multirow[t]{5}{*}{ C } & Subgroup & Pabl & hrgC \\
\hline & EastAsia_sg1 & 35A, F57, F16 & $\mathrm{F} 30$ \\
\hline & EastAsia_sg2 & 51 & 83, ОКз10 \\
\hline & EastAsia_sg3 & F32 & 52 \\
\hline & EastAsia_sg4 & OK113 & \\
\hline
\end{tabular}

b

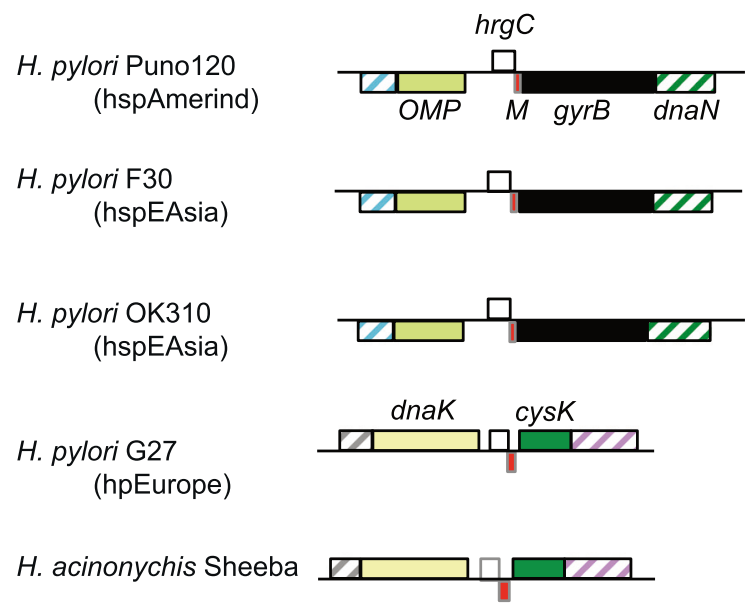

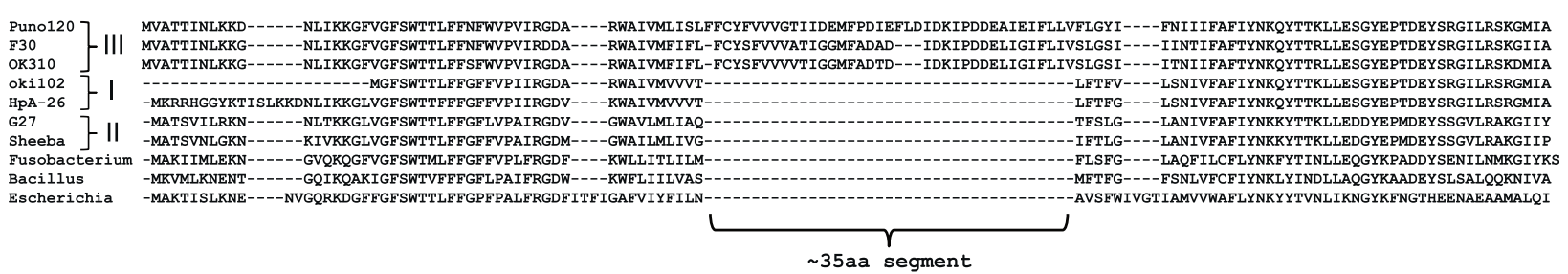

e

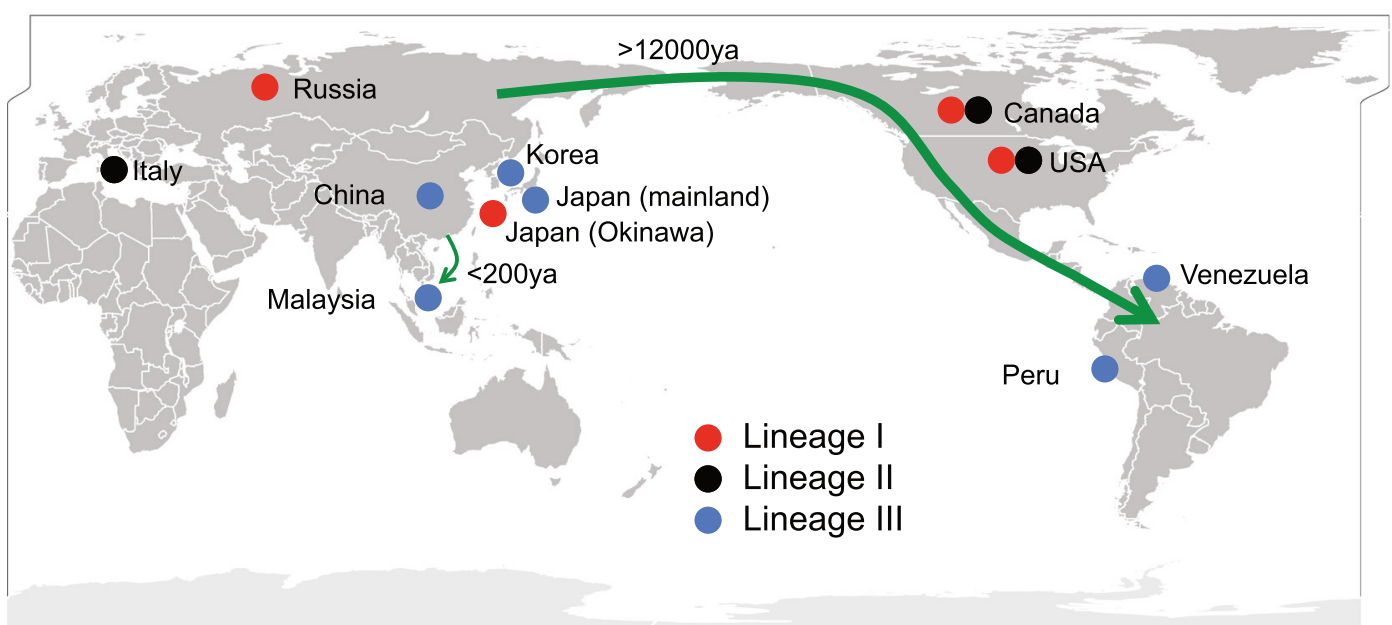

Fig. 4 Replacement of the Pabl family of RM system with hrgC. a Distribution of the Pabl homolog vs. hrgC gene in each population of H. pylori. Only strains that have been completely sequenced and deposited in RefSeq were counted. Analyzed strains are documented in Additional file 2: Table S2. b Genome map comparison around hrgC genes. Orthologous genes are shown as boxes with the same color and pattern. White boxes represent hrgC genes. c Distribution of the Pabl homolog vs. hrgC gene in East Asia subgroups of H. pylori. Only strains reported in Yahara et al. [17] are analyzed. $\mathbf{d}$ Alignment of HrgC proteins. The C-terminal 15 residues of the Escherichia HrgC protein are omitted. Roman numbers right of the $\mathrm{H}$. pylori and $\mathrm{H}$. acinonychis strain names indicate the hrgC lineages shown in Fig. 5. Accession numbers are as follows. Puno120, AEN15115; F30, BAJ56916; OK310, BAM98251; oki102, AHN34853; HpA-14, WP_000301367; G27, ACI26869; Sheeba, YP_665190; Fusobacterium, YP_008019852; Bacillus, EJS64418; Escherichia, AIT14382. e Geographic distribution of hrgC gene. Three lineages of hrgC are colored differently. Green arrows indicate two human migration routes

\section{HrgC phylogeny}

To trace the origin of replacement of the PabI family of the RM system with the $h r g C$ gene, we performed phylogenetic analysis based on the $\mathrm{HrgC}$ protein sequences
(Fig. 5). As expected, all $\mathrm{HrgC}$ proteins from $H$. pylori and $H$. acinonychis clustered together. The most distant lineage included $\mathrm{HrgC}$ proteins encoded at the "pabl" locus and lacking the $\sim 35$ aa segment (lineage I in Figs. 4 and 5). 


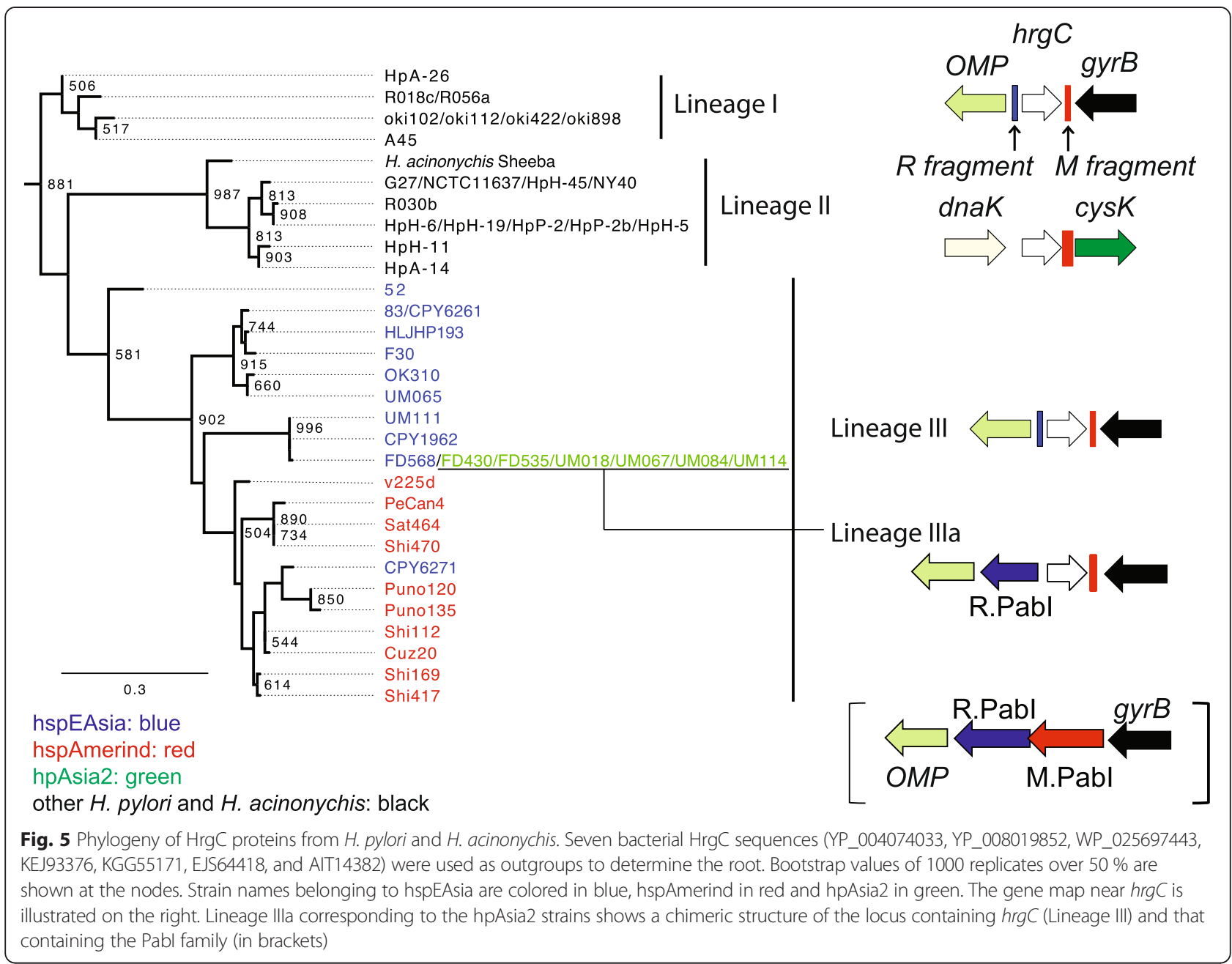

To confirm their distant phylogenetic positions, we generated another phylogenetic tree based on sequences excluding the $\sim 35$ aa segment, with the same topology (data not shown). The phylogenetic position of the HrgC proteins encoded at the " $m c r B$ " locus (lineage II) was unexpected considering their locus different from those of the other lineages (Lineages I and III), both of which are located at the same "pabl" locus, but this position is not well statistically supported.

$\mathrm{HrgC}$ protein sequences from hpAsia2 strains were identical to those from some hspEAsia strains. Among the Malaysian strains, three (UM065, UM111, FD568) from the Malaysian Chinese population belonged to hspEAsia [28, 29]. Five strains (UM018, UM067, UM114, FD430, FD535) from Malaysian Indians and one strain (UM084) from Malays belonged to hpAsia2 (hpSouthIndia) [28, 29]. Two (FD535 and UM084) encoded 208 of 251 aa of the R.PabI homolog besides $\mathrm{HrgC}$ (lineage IIIa in Fig. 5). The remaining four hpAsia2 strains (UM018, UM067, UM114, and FD430) displayed a 124 bp deletion within the R.PabI homolog coding region. These findings collectively suggest horizontal transfer of the $h r g C$ gene from hspEAsia to hpAsia2.

\section{Discussion}

The Pabl family of RM systems displays characteristics similar to other RM systems

The PabI family is unique, encoding a sequence-specific DNA glycosylase/AP lyase as the restriction enzyme $[7,8]$. All other reported RM systems examined to date encode a certain type of DNA phosphodiesterase [9]. Despite fundamental differences in catalytic activity, the PabI family shares many features with other RM systems. Genes for PabI family of restriction glycosylase and adenine methyltransferase genes are tightly associated (Additional file 1: Table S1; Additional file 2: Table S2). Phylogenetic analyses support the theory that that they move together as a unit (Fig. 1). Genome comparisons of closely related strains revealed insertion of the glycosylase and methyltransferase gene pair (Fig. 3). These features are shared with other RM systems. 
Cross-species and cross-genus distribution of orthologous Pabl family of RM systems

Orthologous insertions of the closely related PabI family across species are observed in Helicobacter and Campylobacter species $(\mathrm{H}-1, \mathrm{H}-3, \mathrm{H}-4, \mathrm{H}-9$ and $\mathrm{C}-3$ in Fig. 1, Table 1 and Additional file 3: Figure S1). C-3 represents recent horizontal transfer between species, while $\mathrm{H}-3$ and $\mathrm{H}-9$ possibly indicate insertion in the common ancestor.

Both $\mathrm{H}-3$ in the genus Helicobacter and C-1 in Campylobacter are located downstream of an orthologous gene for GMP synthase. We cannot exclude the possibility that this reflects a preference for the insertion site by the PabI family, but in that case, the underlying molecular mechanism or possible biological significance (such as selective advantage) remains to be established. Recent horizontal transfer between different genera cannot explain the sequence divergence between $\mathrm{H}-3$ and $\mathrm{C}-1$ in Campylobacter sp. MIT 97-5078. The similarity of the C-1 locus in a strain of C. upsaliensis (RM3195) shows a clear case of horizontal transfer from Helicobacter to Campylobacter (Additional file 3: Figure S1). It is likely that the presence of the PabI family downstream of the GMP synthase gene represents an ancient insertion that has been maintained in some strains of these two genera via vertical transmission, possibly coupled with horizontal transfer. Another possibility is an ancient event of horizontal transfer between genera. In our knowledge, there is no report showing long-term maintenance of RM systems leading to their cross-genus distribution. This is the first report of such maintenance. We do not consider it is related to the exceptional catalytic activity of R.PabI homolog, and are convinced that many more cases of long-term maintenance of RM systems be found in the near future.

\section{Replacement of the Pabl family of RM system with an} hrgC gene provides clues on human migration history In some $H$. pylori strains, the PabI family was replaced by an $h r g C$-coding DNA segment. Phylogenetic analysis revealed three $h r g C$ lineages in Helicobacter (Fig. 5, lineages I-III). The history of lineage III is easy to reconstruct (Fig. 4e). A strain of $H$. pylori acquired the $h r g C$ gene that replaced part of the PabI family in East Asia. One of its descendant lineages moved to the Americas, forming the hspAmerind lineage. The uniform presence of $h r g C$ in the hspAmerind lineage is consistent with the isolation of Amerind people in the Americas. In contrast, the other descendant lineage was mixed with other hpEastAsia strains in East Asia, leading to sporadic distribution of the $h r g C$ gene in hspEAsia. Finally, Chinese people moved to Malaysia and the $h r g C$ gene in the hspEAsia strain was transferred to the hpAsia2 strain. This transfer left a fragment of R.PabI homolog, indicating that the replacement is initiated at the M.PabI homolog side (Fig. 5). A previous analysis of $H$. pylori in Southeast Asia revealed that Malaysian hspEAsia strains migrated with Chinese people from Guangzhou and Hong Kong, China within the last 200 years [30]. Thus the transfer of $h r g C$ from hspEAsia to hpAsia2 was a quite recent event. It agrees with the completely identical protein sequences of $\mathrm{HrgC}$ among the hpAsia2 strains. The transfer of DNA segments occurs generally between family members [31]. Thus the presence of $h r g C$ gene in hpAsia2 strains indicates the intermarriage of Chinese immigrant and either Malay or Indian immigrant. The information for such a single event may be lost in the genome-wide study.

In lineage II, the position of $H$. acinonychis Sheeba is the most divergent. The clustering of all $H$. pylori strains is reasonable. The origination of the lineage II $h r g C$ gene remains an open question. Lineages I and III contain the hrgC gene at the "pabI" locus. The simplest explanation is that $h r g C$ gene moved from "pabI" to the " $m c r B$ " locus in the common ancestor of lineage II. Notably, lineage II has the 166 bp fragment of M.PabI homolog, while both I and III contain only the 78 bp fragment of M.PabI homolog (Additional file 5: Figure S3). Thus, the possibility that the PabI family may have once been inserted at the " $m c r B$ " locus and was replaced by $h r g C$ in lineage II cannot be discounted.

Lineage I lacks the $\sim 35$ aa segment at the "pabI" locus. Thus, if replacement of PabI family with $h r g C$ occurred only once, the lineage would retain the original structure. This lineage was obtained from Japan, Russia and North America (Fig. 4e), and their genomes are related to those of hpEurope strains based on the ribosomal protein sequences (NCBI Genome Groups, http://www.ncbi.nlm.nih.gov/ genome/?term=helicobacter\%20pylori). The geographic distribution of lineage I raises the possibility that this sequence was derived from a population in Siberia, and shares the origin with lineage III, which was originated in East Asia.

HrgC was similar to toxins of type I toxin-antitoxin systems in size, with a secondary structure suggestive of a transmembrane helix [26] and apparent mobility between genomes [23]. The toxin-antitoxin and RM systems are analogous from many viewpoints, including attack on host bacteria and gene regulation pathways sometimes involving antisense RNA [32, 33]. The genes encoding defense system components, such as RM, toxin-antitoxin, and CRISPR-Cas systems, typically cluster in islands called "defense islands" [23]. Our findings may indicate stepwise evolution of coupling and cotransfer of RM and toxin-antitoxin systems. More specifically, the promoter for M.PabI homolog may provide an antisense RNA (antitoxin) suppressing the expression of $\mathrm{HrgC}$, a putative toxin. Inhibition of this promoter would lead to attack on the host through postsegregational killing in the PabI family, as well as the 
composite of $\operatorname{hrgC}$ (hypothetical toxin gene) and M.PabI homolog fragment. This may represent takeover of an RM system by a type I toxin. This is just a speculation at present, not supported by any experimental data, but it is worth mentioning to encourage further study. Linkage of the PabI family of the RM system with a toxin gene (Table 1) may have similar significance.

\section{Conclusions}

R.PabI is an exceptional restriction enzyme that acts as a sequence-specific DNA glycosylase. Despite their unique characteristics, R.PabI homologs show similar ways of existence to restriction DNA phosphodiesterases, including co-occurrence and co-mobilization with a methyltransferase (M.PabI homolog) gene, and linkage with the genome rearrangement junctions. These findings are consistent with the theory that the PabI family of RM system is a typical mobile selfish genetic element.

Despite the obvious mobility between genomes, the PabI family of RM system is occasionally observed at the same locus of different strains, species or even genera. This may be attributed to ancient insertion of these RM systems that are maintained via vertical transmission, probably coupled with occasional horizontal transfer via homologous recombination.

The PabI family of RM system is occasionally replaced by an $h r g C$ gene-containing DNA segment in $H$. pylori strains. This substitution possibly occurred in the common ancestor of hspEAsia and hspAmerind. The hspAmerind strains retain this structure owing to geographic isolation, while only a small proportion of hspEAsia strains retain the $h r g C$ gene, indicating that their admixture with strains diverged earlier than the substitution event. In Malaysia, $h r g C$ was horizontally transferred from hspEAsia to hpAsia2 strains. The genetic admixture of $H$. pylori strains would have occurred in an individual or a population, revealing traces of human migration history.

\section{Methods}

\section{Detection of R.Pabl homolog and gene neighborhood} analysis

R.PabI homologs were searched using iterative PSIBLAST [34] with R.PabI as a query until no more proteins were hit over the threshold e-value 0.005 . Once all R.PabI homologs were obtained, the genomic sequences were downloaded from the NCBI website (http://www.ncbi.nlm.nih.gov). At least two annotated genes at each side of R.PabI were obtained manually, and analyzed for methyltransferases.

The orthology of neighboring genes was initially examined based on their annotation, and protein sequences aligned to confirm their sequence identity.
Genome comparison among closely related species/strains TBLASTN search was performed with protein sequences encoded each side of R.PabI homolog genes against genome sequences available on the NCBI website. When both genes were hit to the same genomic sequence of a strain, the relevant sequences were downloaded and analyzed manually. Sequence similarity was visualized with the aid of GenomeMatcher [35]. The ends of sequence identity were determined based on BLASTN results.

\section{Phylogenetic analysis}

Genes of R.PabI and M.PabI homologs, including frameshift mutations or large deletions, were excluded from phylogenetic analysis. HrgC protein sequences hit in the BLASTP search with the $\mathrm{HrgC}$ protein of $H$. pylori strain F30 as a query were downloaded from the NCBI website. Multiple alignments of full-length R.PabI homologs, M.PabI homologs and $\mathrm{HrgC}$ proteins were generated using MAFFT with the linsi option [36]. Phylogenetic analysis was performed on the PhyML server with the LG or WAG model and 1000 bootstrap replicates [37]. The two models resulted in very similar topology in all cases, and therefore only the LG trees were used. A multiple alignment excluding the $\sim 35$ aa segment of $\mathrm{HrgC}$ proteins was also generated with aLRT (alternative likelihood ratio test) values. Trees were drawn using FigTree v1.4.2 (http://tree.bio.ed.ac.uk/software/figtree/).

\section{Comparison of orthologous loci among $\mathrm{H}$. pylori strains} Orthologous loci in $H$. pylori were determined based on the Microbial Genome Database for Comparative Analysis (MBGD) website (http://mbgd.genome.ad.jp/) [38], and examined manually.

\section{Availability of supporting data}

Datasets supporting the results are included within the article and additional files.

\section{Additional files}

Additional file 1: Table S1. R.Pabl and M.Pabl homologs outside $H$. pylori. Accession numbers for fragmented proteins are shown in parentheses. (PDF $83 \mathrm{~kb}$ )

Additional file 2: Table S2. M.Pabl and R.Pabl homologs in the RefSeq genomes of $H$. pylori, $H$. acinonychis and $H$. cetorum. Sheeba, $H$. acinonychis; MIT 99-5656, H. cetorum. All other strains are H. pylori. Strains belonging to hspEAsia are colored in blue and those belonging to hspAmerind in red. In cases where more than two genome sequences are reported for one strain, only one genome is shown. (PDF $86 \mathrm{~kb}$ )

Additional file 3: Figure S1. Intergenic sequences between orthologous M.Pabl homologs and upstream genes. Start codons for the M.Pabl homologs are in bold and stop codons for upstream genes are shaded. Under the nucleotide sequences, encoded protein sequences are shown. (PDF 76 kb) 
Additional file 4: Figure S2. Accumulation of $\mathrm{R}$ and $\mathrm{M}$ genes around the Pabl family of RM system in $\mathrm{H}$. muridarum. The arrows represent genes. Gene numbers are shown above the arrows. The $\mathrm{R}$ and $\mathrm{M}$ gene pairs are indicated by brackets. Target sequences are presented below the RM pairs or M genes. Gene 3185 is a partial duplication of gene 3170 . The sequences of genes 3210 and 3175 showed no significant sequence similarities. (PDF $878 \mathrm{~kb}$ )

Additional file 5: Figure S3. Sequence comparisons of M.Pabl and R.Pabl homolog fragments. Sequences of $H$. acinonychis Sheeba and $H$. pylori G27 are in the " $m c r B$ " locus, while other sequences are in the "pabl" locus. The sequences that do not correspond to M.Pabl or R.Pabl homologs are colored in either red or blue. Nucleotides distinct from the M.Pabl or R.Pabl homolog sequence are shaded in the alignable region. The start codon (TTG) for M.Pabl homolog and stop codon (TAA) for R.Pabl homolog are in bold. (PDF $56 \mathrm{~kb}$ )

\section{Abbreviations}

RM system: Restriction-modification system; AP lyase: Apurinic/apyrimidinic Iyase; 3'-PUA: 3'-phospho alpha, beta-unsaturated aldehyde; ICE: Integrative and conjugative element.

\section{Competing interests}

The authors declare no competing interests.

\section{Authors' contributions}

KKK carried out bioinformatics analysis, interpretation of data, and drafting of the manuscript. IK participated in the study design and coordination as well as preparation of the manuscript. Both authors read and approved the final version of the manuscript.

\section{Acknowledgements}

This work was supported by KAKENHI from the Japanese Society for Promotion of Science or Ministry of Education, Culture, Sports, Science and Technology-Japan [26650123, 15 K14572, 26113704,25291080 to I.K.]. We are grateful to Dr. Ikuo Uchiyama for critical reading of the manuscript.

\section{Author details}

'Department of Computational Biology and Medical Sciences, Graduate School of Frontier Sciences, University of Tokyo, Minato-ku, Tokyo 108-8639, Japan. ${ }^{2}$ Institute of Medical Science, University of Tokyo, Minato-ku, Tokyo 108-8639, Japan. ${ }^{3}$ Genetic Information Research Institute, Los Altos, CA 94022, USA.

Received: 11 June 2015 Accepted: 7 October 2015

Published online: 19 October 2015

\section{References}

1. Loenen WA, Dryden DT, Raleigh EA, Wilson GG, Murray NE. Highlights of the DNA cutters: a short history of the restriction enzymes. Nucleic Acids Res. 2014:42:3-19.

2. Naito T, Kusano K, Kobayashi I. Selfish behavior of restriction-modification systems. Science. 1995;267:897-9.

3. Kobayashi I. Behavior of restriction-modification systems as selfish mobile elements and their impact on genome evolution. Nucleic Acids Res. 2001;29:3742-56.

4. Ishikawa K, Watanabe M, Kuroita T, Uchiyama I, Bujnicki JM, Kawakami B, et al. Discovery of a novel restriction endonuclease by genome comparison and application of a wheat-germ-based cell-free translation assay: Pabl (5'-GTA/C) from the hyperthermophilic archaeon Pyrococcus abyssi. Nucleic Acids Res. 2005:33:e112.

5. Chinen A, Uchiyama I, Kobayashi I. Comparison between Pyrococcus horikoshii and Pyrococcus abyssi genome sequences reveals linkage of restriction-modification genes with large genome polymorphisms. Gene. 2000;259:109-21.

6. Miyazono K, Watanabe M, Kosinski J, Ishikawa K, Kamo M, Sawasaki T, et al. Novel protein fold discovered in the Pabl family of restriction enzymes. Nucleic Acids Res. 2007:35:1908-18.

7. Miyazono K, Furuta Y, Watanabe-Matsui M, Miyakawa T, Ito T, Kobayashi I, et al. A sequence-specific DNA glycosylase mediates restriction-modification in Pyrococcus abyssi. Nat Commun. 2014;5:3178.
8. Fukuyo M, Nakano T, Zhang Y, Furuta Y, Ishikawa K, Watanabe-Matsui M, et al. Restriction-modification system with methyl-inhibited base excision and abasic-site cleavage activities. Nucleic Acids Res. 2015.

9. Orlowski J, Bujnicki JM. Structural and evolutionary classification of Type II restriction enzymes based on theoretical and experimental analyses. Nucleic Acids Res. 2008;36:3552-69.

10. Watanabe M, Yuzawa H, Handa N, Kobayashi I. Hyperthermophilic DNA methyltransferase M.Pabl from the archaeon Pyrococcus abyssi. Appl Environ Microbiol. 2006;72:5367-75.

11. Humbert O, Salama NR. The Helicobacter pylori HpyAXII restrictionmodification system limits exogenous DNA uptake by targeting GTAC sites but shows asymmetric conservation of the DNA methyltransferase and restriction endonuclease components. Nucleic Acids Res. 2008;36:6893-906.

12. Suerbaum S, Josenhans C. Helicobacter pylori evolution and phenotypic diversification in a changing host. Nat Rev Microbiol. 2007:5:441-52

13. Yahara K, Kawai M, Furuta Y, Takahashi N, Handa N, Tsuru T, et al. Genomewide survey of mutual homologous recombination in a highly sexual bacterial species. Genome Biol Evol. 2012;4:628-40.

14. Falush D, Wirth T, Linz B, Pritchard JK, Stephens M, Kidd M, et al. Traces of human migrations in Helicobacter pylori populations. Science. 2003:299:1582-5.

15. Moodley Y, Linz B, Yamaoka Y, Windsor HM, Breurec S, Wu JY, et al. The peopling of the Pacific from a bacterial perspective. Science. 2009;323:527-30.

16. Linz B, Balloux F, Moodley Y, Manica A, Liu H, Roumagnac P, et al. An African origin for the intimate association between humans and Helicobacter pylori. Nature. 2007;445:915-8.

17. Yahara K, Furuta Y, Oshima K, Yoshida M, Azuma T, Hattori M, et al. Chromosome painting in silico in a bacterial species reveals fine population structure. Mol Biol Evol. 2013;30:1454-64.

18. Skarp-de Haan CP, Culebro A, Schott T, Revez J, Schweda EK, Hanninen ML, et al. Comparative genomics of unintrogressed Campylobacter coli clades 2 and 3. BMC Genomics. 2014;15:129.

19. Fischer W, Breithaupt U, Kern B, Smith SI, Spicher C, Haas R, et al. A comprehensive analysis of Helicobacter pylori plasticity zones reveals that they are integrating conjugative elements with intermediate integration specificity. BMC Genomics. 2014;15:310.

20. Kersulyte D, Rossi M, Berg DE. Sequence divergence and conservation in genomes of Helicobacter cetorum strains from a dolphin and a whale. PLoS One. 2013;8, e83177.

21. Furuta Y, Kawai M, Yahara K, Takahashi N, Handa N, Tsuru T, et al. Birth and death of genes linked to chromosomal inversion. Proc Natl Acad Sci U S A. 2011;108:1501-6.

22. Fukuda E, Kaminska KH, Bujnicki JM, Kobayashi I. Cell death upon epigenetic genome methylation: a novel function of methyl-specific deoxyribonucleases. Genome Biol. 2008;9:R163.

23. Makarova KS, Wolf YI, Koonin EV. Comparative genomics of defense systems in archaea and bacteria. Nucleic Acids Res. 2013;41:4360-77.

24. Ando T, Wassenaar TM, Peek Jr RM, Aras RA, Tschumi Al, van Doorn LJ, et al. A Helicobacter pylori restriction endonuclease-replacing gene, hrgA, is associated with gastric cancer in Asian strains. Cancer Res. 2002;62:2385-9.

25. Cole C, Barber JD, Barton GJ. The Jpred 3 secondary structure prediction server. Nucleic Acids Res. 2008;36:W197-201.

26. Park SJ, Son WS, Lee BJ. Structural overview of toxin-antitoxin systems in infectious bacteria: a target for developing antimicrobial agents. Biochim Biophys Acta. 1834;2013:1155-67.

27. Kruger T, Wild C, Noyer-Weidner M. McrB: a prokaryotic protein specifically recognizing DNA containing modified cytosine residues. EMBO J. 1995;14:2661-9.

28. Rehvathy V, Tan MH, Gunaletchumy SP, Teh X, Wang S, Baybayan P, et al. Multiple Genome Sequences of Helicobacter pylori Strains of Diverse Disease and Antibiotic Resistance Backgrounds from Malaysia. Genome Announc. 2013;1:e00687-13.

29. Kumar N, Mariappan V, Baddam R, Lankapalli AK, Shaik S, Goh KL, et al. Comparative genomic analysis of Helicobacter pylori from Malaysia identifies three distinct lineages suggestive of differential evolution. Nucleic Acids Res. 2015;43:324-35.

30. Breurec S, Guillard B, Hem S, Brisse S, Dieye FB, Huerre M, et al. Evolutionary history of Helicobacter pylori sequences reflect past human migrations in Southeast Asia. PLoS One. 2011;6:e22058. 
31. Didelot $X$, Nell S, Yang I, Woltemate $S$, van der Merwe S, Suerbaum S, et al. Genomic evolution and transmission of Helicobacter pylori in two South African families. Proc Natl Acad Sci U S A. 2013;110:13880-5.

32. Mruk I, Liu Y, Ge L, Kobayashi I. Antisense RNA associated with biological regulation of a restriction-modification system. Nucleic Acids Res. 2011;39:5622-32.

33. Mruk I, Kobayashi I. To be or not to be: regulation of restriction-modification systems and other toxin-antitoxin systems. Nucleic Acids Res. 2014:42:70-86.

34. Altschul SF, Madden TL, Schaffer AA, Zhang J, Zhang Z, Miller W, et al. Gapped BLAST and PSI-BLAST: a new generation of protein database search programs. Nucleic Acids Res. 1997;25:3389-402.

35. Ohtsubo Y, Ikeda-Ohtsubo W, Nagata Y, Tsuda M. GenomeMatcher: a graphical user interface for DNA sequence comparison. BMC Bioinformatics. 2008;9:376.

36. Katoh K, Standley DM. MAFFT multiple sequence alignment software version 7: improvements in performance and usability. Mol Biol Evol. 2013;30:772-80.

37. Guindon S, Dufayard JF, Lefort V, Anisimova M, Hordijk W, Gascuel O, et al. New algorithms and methods to estimate maximum-likelihood phylogenies: assessing the performance of PhyML 3.0. Syst Biol. 2010;59:307-21.

38. Uchiyama I, Mihara M, Nishide H, Chiba H. MBGD update 2015: microbial genome database for flexible ortholog analysis utilizing a diverse set of genomic data. Nucleic Acids Res. 2015;43:D270-6.

\section{Submit your next manuscript to BioMed Central and take full advantage of:}

- Convenient online submission

- Thorough peer review

- No space constraints or color figure charges

- Immediate publication on acceptance

- Inclusion in PubMed, CAS, Scopus and Google Scholar

- Research which is freely available for redistribution 